\title{
Johannes Keplers Entfernung von der modernen Wissenschaft
}

Dass Johannes Kepler an der Entstehung der modernen Wissenschaft und ihres kulturellen Einflusses maßgeblich beteiligt war, wird gemeinhin als unstrittig angesehen. Kepler brach mit Dogmen, die seit der Antike die Astronomie beherrschten und einer exakten mathematischen Beschreibung der Planetenbewegung im Wege standen. Im Zentrum standen dabei die Postulate der Kreis- und Gleichförmigkeit der Planetenbewegung, die Kopernikus noch irrtümlich seinem heliozentrischen System zugrunde gelegt hatte. Indem Kepler an die Stelle dieser Postulate seine mathematischen Gesetze - für die er vor allem berühmt ist - setzte, gelang ihm ein entscheidender Schritt zur wissenschaftlichen Begründung des Heliozentrismus im Besonderen und der Astronomie im Allgemeinen. Kepler fragte als einer der ersten nach den physikalischen Ursachen der Planetenbewegung und bereitete damit einer Naturforschung den Boden, in der transzendente Wesenheiten keine Rolle mehr spielten. Auch in anderen Hinsichten wirkte er wegweisend für die methodische Entwicklung der exakten Wissenschaften. Die Verfahren, mit denen er seine Theorie der Beschreibung und Erklärung der Phänomene mit einer großen Anzahl von gemessenen Beobachtungen abglich, setzten beispielhaft empirische Erfahrung als kritische Instanz der Naturforschung ein.

Die Beziehungen von Keplers Werk zur modernen Wissenschaft beschränken sich also keineswegs auf die heute immer noch gültigen Gesetze der Planetenbewegung. Sie sind grundsätzlicher Art und betreffen die Fundamente der Naturforschung. Kepler hatte Anteil an einer wissenschaftlichen Revolution, die sich zu Beginn der Neuzeit vollzog und die sich in vielfältiger Weise mit dem heute vorherrschenden Wissenschaftsverständnis verbinden lässt. Kepler in diesem Sinn als Wegbereiter einer neuen Wissenschaftsauffassung zu sehen, heißt ihn aus heutiger Perspektive zu betrachten. Ist es eine durchaus zulässige Herangehensweise, nach den Ursprüngen der heutigen Wissenschaft und des mit ihr verbundenen Weltbildes zu fragen, kann es vom Standpunkt dieses Interesses aber zugleich problematisch werden, diejenigen Arbeiten Keplers wahrzunehmen, die sich nicht in einen ähnlichen Zusammenhang mit der Gegenwart bringen lassen. Verlorengehen könnten Dimensionen seines Werkes, deren Bedeutung sich weniger vor dem Hintergrund des heutigen als vielmehr des damaligen Wissens erschließt. 
In der Tat war Kepler nicht nur einer der Väter der modernen Wissenschaft, sondern auch ein Renaissance-Humanist, der sich in seinen Forschungen antikem und christlichem Gedankengut verpflichtet sah. Seine Neuerungen vollzogen sich in einem Rahmen traditioneller Orientierungen, in dem das, was der heutige Betrachter als revolutionäre Hauptsache wertet, mitunter nur als beiläufige Erkenntnis vorkommt oder sogar in seiner innovativen Natur verkannt wurde. Ich glaube nicht, dass der grundlegende Charakter einer wissenschaftlichen Neuerung nur in historischer Distanz deutlich wird ${ }^{1}$ und Kepler deshalb gleichsam keine Chance hatte, die ganze Tragweite seiner neuen Astronomie zu erkennen. Umso erstaunlicher ist es $\mathrm{zu}$ sehen, wie sich die Begründung der modernen Wissenschaft bei ihm in gewissem Grad ohne das Bewusstsein der schon antizipierbaren weiteren Erkenntnisentwicklung vollzieht. In seinen historischen Kontext eingebettet erscheint Kepler - vereinfacht gesprochen - weniger revolutionär als aus einer Perspektive, die in seinem Werk vor allem das heute noch Bedeutsame zu finden sucht.

Die bloße Beschränkung auf Keplers historische Mitwirkung bei der Schaffung von Voraussetzungen für die moderne Wissenschaft hat allerdings noch eine weitere Schwierigkeit zur Folge, die zu der eben genannten Problematik invers ist. So wie Keplers traditionelle Orientierungen aus dem Blick geraten können, wenn sein Werk einseitig aus heutiger Perspektive rezipiert wird, lässt sich umgekehrt auch die heutige Wissenschaft einseitig aus der historisch beschränkten Perspektive Keplers betrachten. So kann man diejenigen Elemente der heutigen Wissenschaft besonders schätzen, die auf sich schon Kepler und seine Zeitgenossen stützten. Die verbreitetste Spielart einer solchen - wie ich meine: antiquierten - Betrachtung besteht darin, den frühneuzeitlichen Wahrheitsanspruch der Naturforschung als maßgeblich für die Naturforschung unserer Tage anzusehen. Meines Erachtens unterscheidet sich die Wissenschaft der Gegenwart aber darin von der frühneuzeitlichen Naturforschung, dass sie Erkenntnissen, die nur mit hypothetischem Geltungsanspruch versehen sind, die Wissenschaftlichkeit nicht absprechen muss. ${ }^{2}$ Diese Differenz scheint mir so bedeutsam zu sein, dass man berechtigterweise fragen kann, in welchem Umfang Keplers Wissenschaftsauffassung für die heutige Wissenschaft überhaupt noch typisch ist. Weitere Spielarten antiquierter Lesarten besitzen zwar geringere Relevanz, haben aber dennoch prominente Vertreter gefunden. Wolfgang Pauli und Werner Heisenberg können in diesem Zusammenhang genannt werden. In einigen ihrer Schriften legen sie an die heutige Wissenschaft den Maßstab eines traditionellen, frühneuzeitlichen Weltbildes an.

1 Schiemann 2011, S. 431-453.

2 Schiemann 1997, S. 136-152. 
Die Beurteilung des Verhältnisses zwischen Kepler und der modernen Wissenschaft ist also in verschiedener Weise kontextabhängig. Keplers Wissenschaftsauffassung entfernt sich mit dem Gewicht, das ihren Entstehungsbedingungen zugeschrieben wird, von der modernen Wissenschaftsauffassung. Das Verhältnis beider gestaltet sich ferner unterschiedlich je nach der Auswahl des thematischen Kontextes der Betrachtung. Im Folgenden geht es um die Herausarbeitung von Differenzen zwischen Wissenschaftsauffassungen: nicht nur von der Differenz, die zwischen Keplers und der modernen Auffassung besteht, sondern auch von derjenigen, die sich zwischen seiner Konzeption und den vorangehenden des Mittelalters nachweisen lässt. Dabei wird sich herausstellen, dass Keplers Aufnahme von antikem und christlichem Gedankengut geeignet ist, ihn von der Moderne zu entfernen. Die Thematisierung von Verwandtschaften zwischen den verschiedenen Wissenschaftsauffassungen möchte ich hingegen möglichst gering halten. So werde ich die zweifellos vorhandenen Beziehungen, mit denen sich die Entstehung neuer Wissenschaftsauffassungen vorangegangenen Konzeptionen verdankt, kaum ansprechen. Unberücksichtigt bleibt zum Beispiel die interessante Frage, inwiefern Keplers traditionelle Bindungen zu den Entstehungsbedingungen seines neuen Begriffes von Wissenschaft gehören.

Meine These zur Einordnung Keplers im Spannungsfeld von traditioneller und moderner Wissenschaftsauffassung lautet: Für Keplers naturwissenschaftliche Modernität ist die Kritik qualitativer Differenzen, die Einführung quantitativer Beschreibungen und der methodische Erfahrungsbezug kennzeichnend. Seine Distanz zur modernen Wissenschaftsauffassung tritt hingegen im religiösen Gehalt, im absoluten Geltungsanspruch und im mangelhaften technischen Anwendungsbezug seiner Wissenschaft hervor. Den Ausdruck „modern“ verwende ich in enger und weiter Bedeutung: Im engen Sinn bezeichnet er die für die gegenwärtige Wissenschaft charakteristischen Bestimmungen, im weiten Sinn Kennzeichen der Wissenschaft aus dem historischen Zeitraum, dem auch die gegenwärtige Wissenschaft zuzuordnen ist. Ob diese Zeitspanne bis zum Beginn der Neuzeit zurückreicht, ist eine umstrittene Frage, zu deren Beantwortung die Aufklärung von Keplers Beziehung zur Moderne ein wichtiger Beitrag sein könnte. Die Kontrasteigenschaft $\mathrm{zu}$ „modern“ nenne ich „traditionell“, womit die Bedeutung dieses Ausdrucks auf „vormodern“ eingeschränkt ist. Ausgespart bleiben etwa moderne Traditionsbildungen, ohne die die Moderne sich nicht zu einer Epoche entwickeln kann, nicht aber Traditionen, die, ohne selbst modern zu sein, in der Moderne noch wirksam sind. ${ }^{3}$

3 Von „Moderne“ als Epochenbezeichnung kann auch der Ausdruck „Neuzeit“ unterschieden werden. Die im 16. und 17. Jahrhundert beginnende Neuzeit umfasst nur bestimmte Aspekte der 
Ich werde mit einer kurzen Erinnerung an einige von Keplers Hauptwerken, in denen traditionelle und moderne Elemente eingehen, beginnen (Abschnitt 1). An zwei Beispielen möchte ich dann die Differenz zwischen diesen beiden Elementen näher untersuchen. Das erste Beispiel, Keplers Naturbegriff, dient mir zur Diskussion der Kritik qualitativer Unterscheidungen. Hierbei stehen Keplers Verhältnis zur aristotelischen Naturauffassung und die Relevanz dieser Relation für die moderne Wissenschaftsauffassung im Mittelpunkt (Abschnitt 2). Das andere Beispiel befasst sich mit dem absoluten Wahrheitsanspruch von Keplers Wissenschaft und rückt damit exemplarisch eine Differenz zur modernen Wissenschaftsauffassung in den Vordergrund (Abschnitt 3). Anschließend stelle ich umfassender traditionelle Elemente der frühneuzeitlichen Wissenschaft, wie sie Kepler vertrat, dem modernen Wissenschaftsverständnis gegenüber. Nachdem damit die Entfernung Keplers zur Gegenwart gleichsam maximiert ist, wende ich mich den Wissenschaftsauffassungen von Wolfgang Pauli und Werner Heisenberg $\mathrm{zu}$, die in bemerkenswerter Nähe zu Keplers vormodernen Ansichten stehen und doch ganz im Kontext der Moderne entwickelt wurden (Abschnitt 4). Obwohl also in jüngster Zeit ganz differente Einstellungen zu Keplers Verhältnis zur modernen Wissenschaft vertreten wurden, lässt sich doch eine Tendenz zur Abstandsvergrößerung in dieser Relation ausmachen (Abschnitt 5).

\section{Drei Hauptwerke Keplers}

Meine Darstellung der Entstehung und des Zusammenhangs von Keplers Werken beschränkt sich auf seine wohl bedeutendsten drei Schriften: das „Weltgeheimnis“ (Mysterium cosmographicum) von 1596, der Astronomia Nova von 1609 und die „Fünf Bücher zur Harmonik der Welt“, kurz „Weltharmonik“ (Harmonices Mundi libri V) von 1619. ${ }^{4}$ Im „Weltgeheimnis“ glaubt Kepler den göttlichen Bauplan des Kosmos entdeckt zu haben. Gott habe die Welt mit mathematischer Präzision genau so eingerichtet, wie es im kopernikanischen System erkannt werde. In pythagoreisch-platonischer Tradition erklärt Kepler die Anzahl, Anordnung und Größe der Planetenbahnen durch ein mathematisches Modell, das auf die fünf regulären Polyeder - die sogenannten Platonischen Körper - zurückgreift. Er verschachtelt die Polyeder so ineinander, dass sich in den dadurch entstehenden räumlichen Begrenzungen die damals sechs bekannten Planeten einfügen lassen.

Moderne. Die Entstehung weiterer Bestimmungen der Moderne fokussiert sich meines Erachtens auf das 19. Jahrhundert.

4 Kepler 1596, S. 1-177; ders. 1609; ders. 1619, S. 335-682. 
Mit der Entdeckung eines weiteren Planeten wäre das Modell widerlegt worden. Dass es den Beobachtungen nicht gänzlich widersprach, ist nicht zuletzt den noch unzureichenden Daten geschuldet. Der Wissenschaftshistoriker Owen Gingerich bemerkt dazu: „Niemals in der Geschichte“ hat „ein so falsches Werk [wie das „Weltgeheimnis“] so bahnbrechend die Zukunft der Wissenschaft bestimmt."5

Die Pointe dieser Feststellung besteht darin, dass die traditionsbedingte Fehlerhaftigkeit nicht etwa die Wissenschaft in einer problematischen, sondern in einer äußerst fruchtbaren Weise beeinflusst hat. Das Werk ,verschafft [...] Kepler in der gelehrten Welt den Ruf, ein begabter und überaus gebildeter junger Mann zu sein, auch wenn es neben den lobenden kritische oder gar schmähende Stimmen“ gibt. ${ }^{6} 1597$ gelangt es in die Hände von Tycho Brahe. Brahe findet Keplers Idee bestechend, meint aber zurecht, dass sie durch Beobachtungsdaten besser belegt werden müsse. Kepler teilt diese Ansicht und nimmt 1600 die Einladung Brahes an, sein Assistent zu werden. Brahe gilt als ,der bestausgestattete, zuverlässigste und erfahrenste Astronom seiner Zeit““. ${ }^{7}$ Er verfügt über ein ausgezeichnetes Datenmaterial, das Kepler aber erst nach Brahes überraschendem Tod 1601 vollständig zur Verfügung steht.

Eine mit den damaligen Methoden nur schwer berechenbare Eigenart der Positionsdaten des Planeten Mars bringt ihn auf die in der Astronomia Nova ausgesprochenen These, dass sich dieser Planet wie alle anderen Planeten nicht auf einer kreisförmigen, sondern auf einer elliptischen Bahn bewegt und dass die Bewegung sich geometrisch als nicht gleichförmig beschreiben lässt (erstes und zweites sogenanntes Kepler'sches Gesetz). ${ }^{8}$ Später wird Kepler - neben vielen anderen, heute unbedeutenden Gesetzen - noch ein drittes Gesetz formulieren, das nicht die Bewegungsformen einzelner, sondern den Vergleich verschiedener Planetenbahnen zum Gegenstand hat. Demnach vergrößern sich die Umlaufzeiten mit den Abständen der Planeten zur Sonne $\left(\mathrm{a}^{3} / \mathrm{T}^{2}=\right.$ const). Während die ersten beiden Gesetze nach heutigen Standards der Messtechnik exakt gelten, trifft das dritte Gesetz, auf das ich gleich zurückzukommen werde, nur näherungsweise zu, da es die Masse der Planeten nicht berücksichtigt. Nur wenn man von Keplers bleibenden Beiträgen zur begrifflichen, mathematischen und methodischen Begründung der neuzeitlichen Naturforschung absieht und sich ganz auf seine

5 Gingerich 1993, S. 41.

6 Lemcke 2002, S. 42.

7 Lemcke 2002, S. 47.

8 Für die nichtgleichförmige Bewegung gilt ein strenges Gesetz, das Kepler geometrisch darstellt: Die Verbindungslinie von Sonne und Planet überstreicht in gleichen Zeiten gleiche Flächen, so dass Bahngeschwindigkeit und Sonnenentfernung in umgekehrtem Verhältnis zueinander stehen. 
einzelwissenschaftliche Gesetzeserkenntnis beschränkt, kann man meinen, dass die korrekte theoretische Darstellung der Planetenbahnen [durch diese drei Gesetze ...] die aus der Sicht heutiger exakter Naturwissenschaft [...] einzige seine Wissenschaft überdauernde Erkenntnis gewesen [sei]. ${ }^{9}$

Es ist dieser unhistorischen Perspektive der modernen Naturforschung geschuldet, dass Keplers Lebenswerk ohne die fortgesetzte Geltung seiner Gesetze wohl kaum noch präsent wäre. Sein Ruhm hängt gleichsam an der empirischen Bewährung der von ihm gefundenen mathematischen Beziehungen. ${ }^{10}$

Dabei hat er dieses - von ihm selbst zwar auch als bahnbrechend wahrgenommene - Resultat aber nur als eine aufwendige, wenngleich unvermeidliche Bürde auf dem Weg angesehen, die von Gott geschaffene Ordnung vollständig darzustellen. ${ }^{11}$ Dieser Aufgabe ist die „Weltharmonik“ gewidmet, das dritte Werk, auf das ich zu sprechen kommen möchte. Hier nimmt er den Beweis der vermeintlichen Anzahl der Planeten durch die Platonischen Körper wieder auf, räumt aber ein, dass „die Verhältnisse der Planetenintervalle von der Sonne aus nicht genau von den regulären Körpern allein hergenommen sind“. ${ }^{12}$ In diesem $\mathrm{Zu}$ sammenhang formuliert er sein drittes Gesetz, ${ }^{13}$ das damit korrigierend als Nebenursache auftritt. Statt allein a priori aus den räumlichen Beziehungen zwischen den Platonischen Körpern hergeleitet zu sein, ergeben sich die Abstände der Planeten zur Sonne auch empirisch aus der Messung der Umlaufzeiten. Für Kepler besteht aber die Hauptsache darin, dass die Gesetze der Planetenbewegung und -abstände Teil einer umfassenden metaphysischen Ordnung sind, die sich in musikalischen Beziehungen ausdrücken lässt. Von Gott nach den Gesetzen einer mathematischen Harmonielehre aufgebaut, erscheint der abgeschlossene Kosmos in der „Weltharmonik“ als ein vielstimmiger Gesang himmlischer und irdischer Wesen. Kepler hat seine Weltharmonik als Vollendung seines Lebenswerkes verstanden. In der Vorrede von Buch V heißt es:

Was ich vor 25 Jahren vorausgeahnt hatte [...], was mich veranlasst hatte, den besten Teil meines Lebens, astronomischen Studien zu widmen [...], das habe ich mit Gottes Hilfe [...] endlich ans Licht gebracht. In einem höheren Maße als ich je hoffen konnte, habe ich als

9 Krafft 2005, XXXVIII.

10 Vergleichbares findet man in der Hochschätzung, die anderen Begründern der neuzeitlichen Wissenschaft wie etwa Galileo Galilei, Christian Huygens und Isaac Newton wissenschaftsintern entgegengebracht wird.

11 Vgl. Krafft 2005, XXXVIII.

12 Kepler 1619, S. 586.

13 Kepler 1619, S. 590. 
wahr und richtig erkannt, dass sich die Welt der Harmonik [...] bei den himmlischen Bewegungen findet. ${ }^{14}$

Keplers Werk hatte im 17. Jahrhundert nur einen relativ geringen Einfluss. Seine Erklärung der Planetenbewegung durch magnetische Kräfte wurde - z. B. von Galilei - mit Skepsis betrachtet. Vorübergehend hatte das cartesische Wirbelmodell die größere Anhängerschaft. ${ }^{15}$ Nur die Gesetze der elliptischen Bahnen fanden unter Astronomen Anerkennung und Verwendung. Ihre revolutionäre Kraft entfalteten die Gesetze aber erst, nachdem Newton sie 1686 in den Philosophiae Naturalis Principia Mathematica aus seiner universellen Gravitationstheorie ableitete und dazu nutzte, um irdische und himmlische Bewegungstheorie $\mathrm{zu}$ vereinigen. Was Kepler an Neuerungen zur Begründung der neuzeitlichen Wissenschaft beitrug, beschränkte sich im Wesentlichen auf seine Schriften und deren verspätete Wirkungsgeschichte. Aus diesem Grund ist dem Wissenschaftshistoriker Bernhard Cohen zuzustimmen, dass es nicht angemessen wäre, von einer Kepler'schen Revolution oder einer Kepler'schen Wende der Astronomie zu sprechen, wie dies etwa Martin Carrier und Jürgen Mittelstraß mit Blick auf Keplers fundamentale Kritik der Kreis- und Gleichförmigkeit der Planetenbewegung vorgeschlagen haben. ${ }^{16}$ Im Gegensatz zu Kopernikus brach Kepler zwar mit den antiken und mittelalterlichen Vorgaben, hatte damit aber in seinem Fach keinen durchschlagenden Erfolg. Als hätte er dieses Schicksal vorausgesehen, schreibt er selbstbewusst in der „Weltharmonik“:

Wohlan ich werfe den Würfel und schreibe ein Buch für die Gegenwart oder Nachwelt. Mir ist es gleich. Es mag hundert Jahre seines Lesers harren, hat doch auch Gott sechstausend Jahre [das von Kepler angenommene Alter der Erde] auf den Beschauer gewartet. ${ }^{17}$

Hiermit möchte ich die knappe Erinnerung an Keplers Hauptwerke beschließen. Viele Aspekte seiner Leistungen wie etwa seine optischen Arbeiten oder seine Beiträge zur Erneuerung der Astrologie sind unberücksichtigt geblieben. Meine Darstellung sollte dennoch einen ersten Einblick in die Vielschichtigkeit seiner Werke gegeben haben. Traditionelle und moderne Elemente seiner Kosmologie sind miteinander verwoben, bedingen sich mitunter wechselseitig, stehen aber auch teilweise unverbunden nebeneinander. Traditionell war die Vorstellung eines abgeschlossenen, von Gott geschaffenen und zweckmäßig auf die Bedürfnisse des

14 Kepler 1619, S. 571, vgl. Bialas 2006, S. 120.

15 Cohen, Bernard I. 1994, S. 202.

16 Carrier / Mittelstraß 1989, S. 139 und S. 151.

17 Kepler 1619, zit. nach Bialas 2006, S. 157. 
Menschen eingerichteten Kosmos, d. h. seine religiöse Metaphysik der Wissenschaft; modern nenne ich die mathematische Beschreibung der Planetenbewegung und das Streben nach einer Erklärung, die nicht auf transzendente Wesenheiten zurückgreift. Um Keplers Verhältnis zur Moderne näher zu bestimmen, wende ich mich nun - wie angekündigt - seinem Natur- und Wahrheitsbegriff zu.

\section{Keplers Naturbegriff zwischen Tradition und Moderne}

Keplers Naturbegriff ist exemplarisch für seine Kritik qualitativer Differenzen eine Kritik, die bis heute das Wissenschaftsverständnis prägt. Dieses gut isolierbare moderne Element seiner Wissenschaftsauffassung steht allerdings im unmittelbaren Kontext einer christlichen Orientierung, die ihrerseits von qualitativen Zuschreibungen ausgeht.

Seine „Neue Astronomie“ stellt er ausdrücklich den aristotelischen Prinzipien der Naturlehre gegenüber. In einem Brief an Johann Georg Brengger schreibt er 1607, dass er eine neue „Philosophie oder Himmelsphysik anstelle der HimmelsTheologie oder Metaphysik des Aristoteles“ entwerfen werde. ${ }^{18}$ Aristoteles’ Naturlehre geht von einer doppelten Unterscheidung aus. Die erste Differenz betrifft den Gegensatz zwischen den Bewegungen unter- und oberhalb der Mondbahn, d. h. zwischen sublunarer und supralunarer Welt. Die von der Erde aus gesehen hinter dem Mond befindlichen Sterne (d. h. die Sonne, die Planeten und die heute noch sogenannten Fixsterne) sind mit konzentrischen Kugelschalen verbunden, die durch einen göttlichen Antrieb von außen bewegt werden, so dass sich die Bewegungskraft nach innen fortpflanzt. Oberhalb der Mondbahn wird als natürliche Bewegung die ewige Kreisbewegung angesehen. Darunter, also im irdischen Bereich, kommt hingegen der gradlinigen und nach endlicher Zeit zum Stillstand kommenden Bewegung der Rang der Natürlichkeit zu. Nichtnatürliche, sogenannte gewaltsame Bewegungen, zu denen auf der Erde die kreisförmigen Bewegungen zählen, treten sublunar normalerweise nicht von selbst auf, sondern werden in aller Regel vom Menschen bewirkt. Paradigmatisch dafür ist die Erfindung des Rades. Die natürlichen Bewegungen geschehen hingegen ohne menschliches Zutun. Nach Aristoteles streben die Körper auf und im Umkreis der Erde ihren natürlichen Orten zu, wobei das Leichte gradlinig nach oben aufsteigt und die schweren Körper gradlinig zum Zentrum des Kosmos fallen (weswegen sich seiner Auffassung nach die Erde als Ganze dort befindet).

18 Cohen, Bernard I. 1994, S. 198. 
Die zweite Differenz schließt sich an die erste an und betrifft den Unterschied zwischen natürlichen und künstlichen Bewegungen. Die einen kommen von Natur aus vor, die anderen gehen auf Einwirkungen des Menschen zurück. Aus dieser Unterscheidung entwickelt sich in der Nachfolge von Aristoteles der bis zum Beginn der Neuzeit wirksame Gegensatz von Natur und Technik. Danach ahmt die Technik zwar die Natur nach, vermag aber Phänomene hervorzubringen, die im Prinzip nicht in der Natur vorkommen. Wasser fließt in der Natur bergab, mit Hilfe des Brunnenbaus kann es bergauf fließen. Technik ist in diesem Sinne wider die Natur und kann zur Naturforschung nicht eingesetzt werden.

Kepler bricht mit beiden herkömmlichen Unterscheidungen. Die Bewegungen der Planeten, zu denen er die Erde zählt, führt er auf eine auch auf der Erde wirksame magnetische Kraft zurück. Diese Erklärung ist zwar in sachlicher Hinsicht falsch, hebt aber die Trennung zwischen sub- und supralunarer Welt auf. Keplers Überlegungen weisen bereits in die Richtung eines universellen Kraftgesetzes, wie es später Newton formuliert. Dass Kepler die Unterscheidung von Natur und Technik ebenfalls nicht mehr anerkennt, zeigt die Einbeziehung von technischen Geräten in die Naturforschung. Wie sein Zeitgenosse Galilei setzt er das Fernrohr zur astronomischen Beobachtung ein. Die optische Technik wird zu einem Mittel der Naturbetrachtung, weil sie den gleichen Gesetzen wie die übrige Natur unterliegt. ${ }^{19}$

Obwohl Kepler grundlegende Bestimmungen des aristotelischen Naturverständnisses revidiert, bleibt er ihm doch zugleich verhaftet. Seine fortbestehende antike Orientierung greift vor allem auf diejenigen Elemente zurück, die sich mit seiner christlichen Überzeugung vereinbaren lassen. Nach der vorherrschenden christlichen Lehre war der Kosmos endlich. Die von seinem Zeitgenossen Giordano Bruno und anderen vertretene Auffassung von der Unendlichkeit der Welt weist Kepler unter ausdrücklicher Berufung auf Aristoteles zurück. ${ }^{20}$ Der christlichen Lehre von der durch Gott geschaffenen Natur entspricht die aristotelische Naturteleologie. Alle Dinge der aristotelischen Natur tragen ihren göttlichen Zweck in sich, streben zur Vollendung und sind für den Menschen nützlich. Wie schon bei Kopernikus bleibt bei Kepler die neue Astronomie in ein traditionelles Weltbild eingebettet. Die Erde verliert ihre Mittelpunktstellung, ohne dass schon der herkömmliche Glauben an die Sonderstellung des Menschen im Kosmos erschüttert würde. Doch erste Zweifel werden schon bald nach Keplers Tod etwa von Blaise Pascal formuliert, wenn er schreibt:

19 Vgl. auch Koyré 1980, S. 81.

20 Koyré 1980, S. 64f. Zu Bruno vgl. Blumenberg 1975, S. 416-452. 
Bedenke ich die kurze Dauer meines Lebens, aufgezehrt von der Ewigkeit vorher und nachher; bedenke ich das bißchen Raum, den ich einnehme, und selbst den, den ich sehe, verschlungen von der unendlichen Weite der Räume, von denen ich nichts weiß und die von mir nichts wissen, dann erschaudere ich und staune, dass ich hier und nicht dort bin. ${ }^{21}$

Pascals Schrecken gegenüber der kosmischen Kontingenz und Verlassenheit des Menschen bleibt jedoch zunächst im Diskurs um das Weltbild der neuzeitlichen Wissenschaft bis weit ins 19. Jahrhundert eine Ausnahme. Kennzeichnend ist vielmehr die Traditionslinie der Physikotheologie bzw. Natürlichen Theologie, die in den Naturphänomenen Bestätigung und sogar Beweise für die Existenz Gottes und seiner Eigenschaften findet. Neben Kepler ist dieser Linie auch Newton zuzurechnen. ${ }^{22}$ Erst mit dem 19. Jahrhundert zerbricht die Einheit von religiösem Glauben und Naturforschung, teils als Konsequenz der auf die industrielle Revolution folgenden Säkularisierung, ${ }^{23}$ teils als Resultat von wissenschaftlichen Einsichten wie Pierre Simon Laplace' Fortentwicklung der Himmelsmechanik und Charles Darwins Evolutionslehre - um nur einige der wichtigsten Faktoren zu nennen. ${ }^{24}$

\section{Keplers Wahrheitsbegriff im Kontrast zum modernen Verständnis}

Naturerkenntnis ist nach Kepler ein Wissen von Gottes Schöpfung, weswegen er die Astronomie als „Gottesdienst“ bezeichnet. ${ }^{25}$ Gott habe den Menschen mit Geist ausgestattet, damit er die Welt in ihrer wahren Beschaffenheit erkenne. Wahre Erkenntnis kann Kepler zufolge induktiv aus der Erfahrung durch Beobachtung und Messung gewonnen oder a priori aus der Vernunft abgeleitet werden, bedarf dann aber der Bestätigung durch die Erfahrung. ${ }^{26}$ Der damit formulierte Wahrheitsanspruch gründet sich auf das rationale Vermögen des Subjektes und ist gegen autoritative Bevormundung gerichtet.

Auf die Meinungen der Heiligen aber über diese natürlichen Dinge antworte ich mit dem einzigen Wort: In der Theologie gilt das Gewicht der Autoritäten, in der Philosophie aber das der Vernunftgründe. Heilig ist zwar Laktanz, der die Kugelgestalt der Erde leugnete, heilig

21 Pascal 1978, S. 114, Fragment Nr. 205 nach Brunschvicg; vgl. Koyré 1980, S. 49.

22 Zu Newton vgl. Barbour 2003, S. 39-41.

23 Mortensen 1995, S. 11.

24 Barbour 2003, S. 107-110; Polkinghorne 2008, S. 59.

25 Krafft 2005, XLIX.

26 Bialas 2006, S. 49-64. 
Augustinus, der die Kugelgestalt zugab, aber Antipoden leugnete, heilig das Offizium unserer Tage, das die Kleinheit der Erde zugibt, aber ihre Bewegung leugnet. Aber heiliger ist mir die Wahrheit, wenn ich bei aller Ehrfurcht vor den Kirchenlehrern, aus der Philosophie beweise, dass die Erde rund, ringsum von Antipoden bewohnt, ganz unbedeutend und klein ist und auch durch die Gestirne hin eilt [...]. ${ }^{27}$

Keplers Wahrheitsverständnis ist für die frühneuzeitliche Wissenschaft typisch. Es richtet sich gegen die mittelalterliche Lehre von der doppelten Wahrheit, derzufolge die Wahrheit der Mathematik von der Wahrheit des Glaubens zu trennen ist. Erstere würde nichts über die wirkliche Weltstruktur, die dem menschlichen Erkenntnisvermögen überhaupt verborgen sei, aussagen. Dagegen knüpft Kepler an Platons Naturphilosophie an, die ein Abbild des Wesens der Welt in die Mathematik legt und für erkennbar hält. Platon bestritt allerdings die Möglichkeit einer exakten Naturforschung. Die Erfahrung wäre viel zu unbeständig, als dass sie zum Gegenstand eines Wissens gemacht werden könnte. Erst Aristoteles begründete den Wissenschaftscharakter der Naturforschung, an dem die frühneuzeitliche Revolution der Physik wieder anknüpft. Demnach kann die Naturforschung Wissenschaft sein, weil ihre Erkenntnisse wahr sind und dem Ziel dienen, ein vollständiges Wissen der Welt zu erreichen. Kepler steht auch in dieser Tradition. Seine Gesetze beanspruchen nicht, (platonisch) in einer bloß wahrscheinlichen Beziehung zu den Messungen der beobachteten Bewegungen der Planetenörter zu stehen, sondern diese Messungen selbst exakt darzustellen. Sie haben keinen hypothetischen Status.

Obwohl Wahrheit und nicht Hypothesen das Ziel der wissenschaftlichen Erkenntnis ist, räumt Kepler Hypothesen einen wichtigen Stellenwert in der wissenschaftlichen Methode ein:

Zuerst entwerfen wir uns in den Hypothesen ein Bild von der Natur der Dinge. Dann konstruieren wir, auf sie gestützt, den Calculus, die Berechnungsweise. Wir zeigen damit die Bewegungen auf. Schließlich prüfen wir auf zurücklaufendem Weg die wahren Vorschriften der Berechnung. ${ }^{28}$

Mit dem „zurücklaufenden Weg“ ist die empirische Prüfung der Hypothesen an der Erfahrung gemeint. Dass der Mensch schon vor aller Erfahrung Hypothesen bilden kann, die sich später als richtig herausstellen, führt Kepler auf das Vorhandensein sogenannter Archetypen zurück. So bezeichnet er Urbilder der

27 Kepler 1609, S. 40.

28 Zit. nach Bialas 2006, S. 62. 
Schöpfung, die in der menschlichen Seele präsent sind..$^{29}$ Der Mensch kann aber nicht wissen, ob seine Hypothesen der Wahrheit entsprechen, bevor er sie nicht an der Erfahrung geprüft hat. Wissenschaft zeichnet sich so durch die Überführung von hypothetischer Erkenntnis in wahre Erkenntnis aus.

Das Verhältnis von Hypothese und Wahrheit wurde von den Begründern der neuzeitlichen Wissenschaft unterschiedlich bestimmt. Gemeinsam ist ihnen allerdings, dass die Bildung von wissenschaftlichen Hypothesen, sofern sie überhaupt zugelassen sind, der Wahrheitsfindung zu dienen habe. ${ }^{30}$ Diese Orientierung der Wissenschaftsauffassung am Wahrheitsbegriff begann erst in der zweiten Hälfte des 19. Jahrhunderts, als die Probleme einer vollständigen und ausschließlich gültigen naturwissenschaftlichen Welterkenntnis immer deutlicher hervortraten, an Einfluss zu verlieren. ${ }^{31}$ Ein erster bedeutender Schritt der Relativierung des wissenschaftlichen Wahrheitsanspruches ging auf die Entdeckung unterschiedlicher, aber gleichberechtigter geometrischer Darstellungen des Raumes zurück. ${ }^{32}$ Die Wahrheit der klassischen physikalischen Theorien wurde dann zu Beginn des 20. Jahrhunderts durch die Revolution der modernen Physik, d. h. durch die Relativitätstheorien und die Quantenmechanik, erschüttert. Mit Bezug auf diese Ereignisse hat der Wissenschaftsbegriff der Physik ganz neue Dimensionen in sich aufgenommen. Wissenschaftliche Theorien haben ihren Wahrheitsanspruch auf die Voraussage von Phänomenen beschränkt und ihren physikalischen Erklärungen einen bloß noch hypothetischen Charakter gegeben. Sie haben auf einen ausschließlichen Geltungsanspruch verzichtet und die Möglichkeit von unterschiedlichen, aber empirisch äquivalenten Darstellungen eingeräumt. Vor dem Hintergrund dieser Entwicklung kann man Keplers Wahrheitsund Wissenschaftsverständnis kaum als modern bezeichnen.

\section{Moderne Wissenschaft}

Mit dieser Feststellung bin ich schon dazu übergegangen, traditionelle Elemente von Keplers Wissenschaftsbegriff dem modernen Verständnis gegenüberzustellen. ${ }^{33} \mathrm{Im}$ Gegensatz zur frühen Neuzeit sind wissenschaftliche und religiöse Er-

29 Bialas 2006, S. 51-59 und S. 121.

30 Vgl. McMullin 2009.

31 Eine gewichtige gegenläufige Tendenz stellen die Einflüsse des englischen Empirismus auf das Wissenschaftsverständnis des Mechanismus dar. Vgl. Schiemann (1997, S. 50).

32 Schiemann 1997, S. 219-234.

33 Die Beziehungen von frühneuzeitlicher und moderner Wissenschaft stelle ich idealtypisch dar. Bei der Kennzeichnung des Wissenschaftsbegriffes rekurriere ich vor allem auf den Diskurs 
kenntnis in der Moderne völlig auseinandergetreten. Alle Vorstellungen des Einflusses transzendenter Wesenheiten auf die objektive Erfahrung oder einer planmäßig eingerichteten Zweckmäßigkeit der beobachtbaren Phänomene sind aus dem Naturbild der Naturwissenschaften entfernt worden. ${ }^{34}$ Die Erkenntnis der Wissenschaft hat durch ihr technisch vermitteltes Vordringen in die Dimensionen des ganz Großen und des ganz Kleinen ferner jede Anschaulichkeit verloren. Stand für Kepler mit der Sonne noch der Inbegriff der Sichtbarkeit im Zentrum des Kosmos, bilden heute nicht anschaulich nachvollziehbare Auswertungen spektroskopischer Daten den Bezugspunkt von kosmologischen Theorien. Sie lehren uns, dass der Kosmos kein Zentrum hat, seine Ausdehnung alle Vorstellungskraft sprengt und einer sich unaufhaltsam beschleunigenden Expansion unterworfen ist. ${ }^{35}$ Keplers heliozentrische Theorie von der Abgeschlossenheit des Kosmos können wir in ihren wesentlichen Aspekten als falsifiziert ansehen. Sie widerspricht kosmologischen Daten. Die mit diesen Daten verträglichen Theorien (z. B. des Urknalls oder der Expansion des Universums) haben trotz ihrer bloß hypothetischen Geltung große Überzeugungskraft. ${ }^{36}$

Die Hypothetizität der Erkenntnis ist in den Wissenschaften nicht nur zugelassen, sondern außerdem zum Kriterium der Wissenschaftlichkeit geworden. Insofern wissenschaftliche Erkenntnis immer für Kritik offen und revisionsfähig bleibt, hat sie keinen abschließenden, sondern immer nur vorübergehenden Charakter. Es kann zwar in den Wissenschaften weiterhin - ähnlich wie zu Keplers Zeiten - nach einem vollständigen und ausschließlich geltenden System der Erkenntnis gestrebt werden. Doch diese Zielsetzung ist weniger Gegenstand eines wissenschaftlichen Diskurses, als dass sie in die eher privaten Motivationen von Forschern und Forscherinnen eingeht. ${ }^{37}$

In den Wandel des Wissenschaftsbegriffes hat als weiteres signifikantes Merkmal eine zunehmende Anwendungsrelevanz der wissenschaftlichen Erkenntnis Eingang gefunden. Wissenschaftliche Erkenntnisse dringen in immer mehr gesellschaftliche Bereiche ein, beweisen dort ihre Nützlichkeit und werden

der Wissenschaftstheorie, d. h. der Philosophinnen und Philosophen, die sich mit der Wissenschaft befassen, der Wissenschaftsgeschichte und der Wissenschaftlerinnen und Wissenschaftler, die über ihre eigene Arbeit reflektieren. Einführend in die Wissenschaftsgeschichte der Neuzeit sind Cohen, Bernard I. 1994 und Cohen, H. Floris 1994, in die Wissenschaftstheorie der Gegenwart Bartels 2007, und Carrier 2006.

34 Barbour 2003, S. 233-348.

35 Rees 2003, S. 114-135; Silk 2006, S. 180-193.

36 Die Hypothetizität der kosmologischen Theorien geht teils auf die Geltungsunsicherheit aufgrund fehlender Daten zurück, teils hängt sie mit den strukturellen Bedingungen der wissenschaftlichen Erkenntnisbildung zusammen.

37 Schiemann 2012. 
für die Aufrechterhaltung der Lebensverhältnisse unverzichtbar. $\mathrm{Zu}$ den kennzeichnenden Einsichten der modernen Wissenschaft gehört, dass man die durch sie ermöglichten Techniken auch dann zu menschlichen Zwecken einsetzen kann, wenn man die zugrundeliegenden Mechanismen noch nicht verstanden hat. ${ }^{38}$ Gegenüber diesem Zurücktreten der Wahrheit vor der Technik wird noch einmal die Differenz zu Keplers Wissenschaftsverständnis deutlich. Kepler kannte ebenfalls Anwendungen seiner astronomischen Erkenntnis: Er bestritt zeitweise einen Teil seines Lebensunterhaltes mit astrologischen Arbeiten. Aber Astrologie ist keine materielle Technik zum Vorteil des Menschen. Zudem verstand Kepler die Wissenschaft als reine theoretische Erkenntnis, völlig unabhängig von aller Nutzanwendung:

Unser Bildner hat zu den Sinnen den Geist gefügt, nicht bloß, damit sich der Mensch seinen Lebensunterhalt erwerbe - das können viele Arten von Lebewesen mit ihrer unvernünftigen Seele viel geschickter -, sondern auch dazu, dass wir vom Sein der Dinge, die wir mit Augen betrachten, zu den Ursachen ihres Seins und Werdens vordringen, wenn auch weiter kein Nutzen damit verbunden ist. ${ }^{39}$

Dass sich seit Keplers Zeit der Wissenschaftsbegriff gewandelt hat, heißt nun aber nicht, dass traditionelle Vorstellungen im Verständnis der Wissenschaft keine Rolle mehr spielen. Wie das Wahrheitsstreben als private Motivation immer noch Motor von Erkenntnisbemühungen sein kann, vermögen metaphysische Ordnungsvorstellungen in die Überzeugungen der Forscherinnen und Forscher einzugehen. Als Beispiel dafür möchte ich abschließend auf die Wissenschaftsauffassung zweier Vertreter der modernen Physik - Wolfgang Pauli und Werner Heisenberg - zu sprechen kommen.

Beide Physiker gehören $\mathrm{zu}$ den herausragenden Begründern der heutigen atomphysikalischen Theorien. Mit ihren Arbeiten haben sie zur Formulierung der Quantenmechanik entscheidend beigetragen. Pauli und Heisenberg, die sich während des Studiums 1920 in München kennengelernt hatten, verband eine lebenslange, nicht immer ungetrübte Freundschaft. Ihre Wissenschaftsauffassungen berühren sich in ihren positivistischen Überzeugungen, wie sie in der von ihnen vertretenen Deutung der Quantenmechanik zum Ausdruck kommen. In seinem Text „Der Einfluß archetypischer Vorstellungen auf die Bildung naturwissenschaftlicher Theorien bei Kepler“ beschäftigte sich Pauli intensiv mit Keplers Vorstellungen. ${ }^{40}$ Darin schließt er sich Keplers Lehre von den schon er-

38 Schiemann 2011.

39 Kepler 1595, S. 7.

40 Pauli 1995, S. 295-300. 
wähnten Archetypen an, die die Bestimmungen der Welt als mathematische Beziehungen enthalten und in die Seelen der Menschen eingelassen sind. Sie garantieren die einheitliche Struktur der Welt und ermöglichen die wissenschaftliche wie die intuitive Naturerkenntnis. Bei Pauli verbindet sich dieser Rückgriff auf mystizistisches Gedankengut mit einer Kritik an der Moderne, in der es „fremd geworden“ sei, „in lebendiger Beziehung zum Gesamtwissen der Zeit und zum tatsächlichen Prozess der Erkenntnis [zu] stehen“.41

Heisenberg sprach den Archetypenbegriff nur vereinzelt an, vertrat aber eine Paulis Vorstellung verwandte Position. Seiner Überzeugung nach liegt der Wirklichkeit und ihrer zeitlichen Veränderung die Dynamik einer ,zentrale[n] Ordnung“ zugrunde, an der einzelne ausgewählte Menschen in ihrer - sei es wissenschaftlichen oder künstlerischen - Erkenntnis teilhaben. ${ }^{42}$ Bemerkenswerterweise muss die Struktur der Welt aber keine durchgängige Einheit aufweisen. Sie hat einen pluralen Charakter, hängt von den subjektiven und objektiven Erkenntnisbedingungen der Menschen ab und unterliegt einem Wandel, in dem Veränderungen sprunghaft auftreten können. Auch Heisenberg verbindet seine spekulative Weltsicht mit einer Kritik, die sich unter anderem gegen einen ausschließlich geltenden Wahrheitsanspruch der Naturwissenschaften wendet. ${ }^{43}$

Bei beiden Physikern bilden traditionelle und moderne Vorstellungen ein Spannungsverhältnis, ergänzen sich aber dennoch zu einer Weltsicht, die den in der Gegenwart erreichten Verlust an unmittelbarer Erfahrung aufzuheben versucht. Im Gesamtwerk von Heisenberg und Pauli machen die spekulativen Überlegungen nur einen kleinen Teil aus. Ihr untergeordnetes Auftreten in den Schriften steht in keinem Verhältnis zum Umfang, den die nichtempirische Erkenntnis in Keplers Texten einnimmt. Heisenberg thematisiert diese Differenz am Beispiel einer Schrift von Kepler sogar ausdrücklich. In einem Sammelband mit dem Titel Das Naturbild der Physik veröffentlicht er 1955 neben eigenen Aufsätzen auch historische Quellen, die mit kleinen - vermutlich von ihm selbst verfassten Kommentaren versehen sind. Die erste Quelle besteht aus Auszügen aus dem „Weltgeheimnis“. Im Kommentar dazu heißt es:

Zwei Dinge springen dem heutigen Leser, der mit den modernen Naturwissenschaften ganz bestimmte Vorstellungen verbindet, in die Augen:

1. Die Naturwissenschaften sind für KEPLER ganz und gar nicht Mittel, die dem materiellen Nutzen des Menschen dienen, die ihm die Technik ermöglichen, mit deren Hilfe er sich besser in der unvollkommenen Welt einrichten kann, und die ihm den Weg des Fortschritts eröffnen. Die Naturwissenschaften sind ihm im Gegenteil ein Mittel zur Erhebung des Geistes, ein Weg

41 Pauli 1995, S. 300.

42 Heisenberg 1971, S. 43-51; Heisenberg 1922, S. 207-226; vgl. Schiemann 2008, S. 78-83. 43 Schiemann 2008, S. $91 \mathrm{f}$. 
Ruhe und Trost zu finden im Anschauen der ewigen Vollkommenheit der Schöpfung.

2. Eng in Zusammenhang damit steht die erstaunliche Verachtung des Empirischen. Die Erfahrung ist nur das zufällige Finden von Zusammenhängen, die auf Grund der Einsicht in die apriorischen Gründe viel zuverlässiger erfaßt werden können. Die vollkommene Übereinstimmung zwischen der Ordnung der "Sinnesdinge“ - die Werke Gottes sind - und den mathematischen und intelligiblen Gesetzen - den $<$ Gedanken $>$ Gottes - wird zum Grundgedanken der ,Harmonices Mundi ${ }^{4}{ }^{44}$

Mit der ersten Bemerkung erkennt Heisenberg als Auswirkung von Keplers kontemplativer Wissenschaftsauffassung eine der maßgeblichen Differenzen zur modernen Wissenschaft: Keplers Wissenschaft geht der technische Anwendungsbezug ab. Zwischen dem praktischen Erkenntnisnutzen der Moderne und den von Kepler verfolgten ideellen Interessen postuliert Heisenberg zu Recht einen Gegensatz. So stark diese Differenz aber sein mag, rechtfertigt sie nicht Heisenbergs zweite Bemerkung, mit der er Keplers Wissenschaft den systematischen Erfahrungsbezug abspricht. Umgekehrt verdankt die moderne Naturforschung Kepler das Vorbild einer zielstrebigen Datensuche und -auswertung der Planetenbewegung. Freilich ist Heisenberg wieder zuzustimmen, wenn er darauf hinweist, dass Kepler die „vollkommene Übereinstimmung zwischen der Ordnung der ,Sinnesdinge““, zu der Daten zählen, und „mathematischen Gesetzen“ beanspruchte. Mit der Moderne teilt Kepler, dass die Übereinstimmung exakt sein soll, gegen sie meint er mit „Vollkommenheit“ auch die Unumstößlichkeit dieser Passung.

Heisenberg weiß um den bahnbrechenden Beitrag, den Kepler zur Begründung der modernen Wissenschaft geleistet hat, und bringt zugleich die Differenz, die zwischen der Wissenschaftsauffassung von Kepler und derjenigen heutiger Leser besteht, in Anschlag. Eben diese Vorstellung, dass heute gültige Erkenntnisse im Kontext von aus heutiger Sicht fragwürdigen Wissenschaftsauffassungen entstanden, ist für das moderne Verständnis der Wissenschaft bezeichnend und wäre für Kepler selbst wohl nicht nachvollziehbar gewesen..$^{45}$

\section{Schluss}

Man hat die Neubegründung der Wissenschaft im 16. und 17. Jahrhundert als eine verschiedene Disziplinen umfassende Revolution charakterisiert und davon

44 Heisenberg 1955, S. 58f. Hervorheb. im Orig.

45 Im modernen Wissenschaftsverständnis wird systematisch zwischen Rechtfertigungs- und Entdeckungskontext getrennt. Vgl. Schiemann 2005, S. 23-39. 
grundlegende Änderungen der wissenschaftlichen Erkenntnis, die sich auf die Konzeptionen und den Gegenstandsbereich einer Disziplin beschränken, abgehoben. ${ }^{46}$ Mit dieser begrifflichen Unterscheidung kann man sagen, dass Kepler zur neuzeitlichen Revolution der Wissenschaft beigetragen hat, ohne eine Revolution in der Astronomie bewirkt zu haben. Seine Modernität besteht vornehmlich in seinem Anteil an der Schaffung fundamentaler begrifflicher und methodischer Neuerungen der Naturforschung seiner Zeit. Begrifflich werden qualitative Differenzen wie die traditionelle von Natur und Technik abgelehnt und vereinheitlichende physikalische Größen wie die der Kraft in engem Bezug zur Erfahrung eingeführt; methodisch wird die Mathematisierung durch quantitative Beschreibungen sowie deren empirische Begründ- und Überprüfbarkeit vorangebracht.

Doch in der modernen Naturwissenschaft unserer Tage zählen allgemeine historische Voraussetzungen weniger als bewährte spezielle Gesetzesgeltungen. Deshalb kann man vermuten, dass sich Keplers wissenschaftsimmanente Bekanntheit vor allem der andauernden Geltung der von ihm aufgestellten Gesetze verdankt. Zwar versteht sich die wissenschaftliche Erkenntnis in der Moderne als jederzeit und grundlegend revisionsfähig, doch schätzt sie gerade diejenigen Erkenntnisse, die bisher allen - vielleicht bloß denkbaren - Revisionsversuchen widerstanden haben. Allerdings sind Keplers Gesetze heute bloß noch ein Spezialfall der kosmischen Gravitationswechselwirkungen, die durch Albert Einsteins allgemeine Relativitätstheorie zur Zeit am besten beschrieben werden. Begriff Kepler seine Gesetze als integralen Bestandteil einer die Natur einbeziehenden göttlichen Ordnung, beschreiben sie heute den nur vorübergehend stabilen $\mathrm{Zu}$ stand der Planetenbewegung in einem Universum, von dem mit guten Gründen behauptet wird, dass es irreversibel zur Auflösung jeglicher Ordnungsstruktur tendiert. Es ist nicht leicht, von dieser ziemlich trostlosen Aussicht, die keinen Glauben an Transzendenz mehr voraussetzen muss, eine Brücke zum religiösen Glauben zu schlagen, der für Kepler alle Wissenschaft und ihre Wahrheit begründete.

\section{Literatur}

Barbour, Ian G. (2003): Wissenschaft und Glaube. Historische und zeitgenössische Aspekte. Göttingen: Vandenhoeck \& Ruprecht.

Bartels, Andreas/Stöckler, Manfred (Hrsg.)(2007): Wissenschaftstheorie. Ein Studienbuch. Paderborn: Mentis.

Bialas, Volker (2006): Johannes Kepler. München: Beck.

46 Cohen, Bernard I. 1994, S. 129-164; Schiemann 2011. 
Blumenberg, Hans (1975): Die Genesis der kopernikanischen Welt. Frankfurt am Main: Suhrkamp.

Carrier, Martin/Mittelstraß, Jürgen (1989): „Johannes Kepler“. In: Böhme, Gernot (Hrsg.): Klassiker der Naturphilosophie. Von den Vorsokratikern bis zur Kopenhagener Schule, München: Beck, S. 137-157.

Carrier, Martin (2006): Wissenschaftstheorie. Hamburg: Junius.

Cohen, Bernard I. (1994): Revolution in der Naturwissenschaft. Übersetzt von Kutschmann, Werner. Frankfurt am Main: Suhrkamp.

Cohen, H. Floris (1994): The scientific revolution: a historiographical inquiry. Chicago: University of Chicago Press.

Gingerich, Owen (1993): The Eye of Heaven. Ptolemy, Copernicus, Kepler. New York: American Institute of Physics.

Heisenberg, Werner (1922): „Das Naturbild Goethes und die technisch-naturwissenschaftliche Welt“. In: Ders. (Hrsg.): Schritte über Grenzen, München: Piper, S. 207-226.

Heisenberg, Werner (1955): Das Naturbild der heutigen Physik. Hamburg: Rowohlt.

Heisenberg, Werner (1971): „Wolfgang Paulis philosophische Auffassungen“. In: Ders. (Hrsg.): Schritte über Grenzen, München: Piper, S. 43-51.

Kepler, Johannes (1596): „Weltgeheimnis (Mysterium cosmographicum)“. In: Ders. (Hrsg): Was die Welt im Innersten zusammenhält. Antworten aus Keplers Schriften, Wiesbaden: Matrix, S. 1-177.

Kepler, Johannes (1609): Astronomia Nova. Neue, ursächlich begründete Astronomie oder Himmelsphysik. Wiesbaden: Matrix.

Kepler, Johannes (1619): „Weltharmonik (Harmonices Mundi Libri V)“. In: Ders. (Hrsg): Was die Welt im Innersten zusammenhält. Antworten aus Keplers Schriften, Wiesbaden: Matrix, S. $335-682$.

Koyré, Alexandre (1980): Von der geschlossenen Welt zum unendlichen Universum. Frankfurt am Main: Suhrkamp.

Krafft, Fritz (2005): „Johannes Kepler - Die neue, ursprünglich begründete Astronomie“. In: Kepler, Johannes: Astronomia Nova. Neue, ursächlich begründete Astronomie oder Himmelsphysik. Wiesbaden: Matrix.

Lemcke, Mechthild (2002): Johannes Kepler. Hamburg: Rowohlt.

McMullin, Ernan (2009): „Hypothesis in early modern science“. In: Heidelberger, Michael/Schiemann, Gregor (Hrsg.): The Significance of the Hypothetical in the Natural Sciences, Berlin u. a.: de Gruyter, S. 7-38.

Mortensen, Viggo (1995): Theologie und Naturwissenschaft. Gütersloh: Gütersloher Verlagshaus.

Pascal, Blaise (1978): Über die Religion und über einige andere Gegenstände. Übers., hg. und mit Nachwort von Ewald Wasmuth. Frankfurt am Main: Schneider.

Pauli, Wolfgang (1995): „Der Einfluss archetypischer Vorstellungen auf die Bildung naturwissenschaftlicher Theorien bei Kepler“. In: Atmanspacher, Harald u. a. (Hrsg.): Der Pauli-Jung-Dialog und seine Bedeutung für die moderne Wissenschaft, Heidelberg: Springer, S. 295-300.

Polkinghorne, John (2008): „Christianity and Science“. In: Clayton, Philip/Simpson, Zachary (Hrsg): The Oxford Handbook of Religion and Science, Oxford: University Press.

Rees, Martin (2003): Das Rätsel unseres Universums: Hatte Gott eine Wahl? München: Beck. 
Schiemann, Gregor (1997): Wahrheitsgewißheitsverlust. Hermann von Helmholtz' Mechanismus im Anbruch der Moderne. Eine Studie zum Übergang von klassischer zu moderner Naturphilosophie. Darmstadt: Wissenschaftliche Buchgesellschaft.

Schiemann, Gregor (2005): „Inductive Justification and Discovery. On Hans Reichenbach's Foundation of the Autonomy of the Philosophy of Science“. In: Schickore, Jutta/Steinle, Friedrich (Hrsg.): Revisiting Discovery and Justification. Dordrecht: Kluwer, S. 23-39.

Schiemann, Gregor (2008): Werner Heisenberg. München: Beck.

Schiemann, Gregor (2011): „Is an epoch-making change in the development of science currently taking place? A critique of the ,epochal-break-thesis““. In: Carrier, Martin/Nordmann, Alfred (Hrsg.): Science in the Context of Application, Berlin: Springer, S. 431- 453.

Schiemann, Gregor (2012): „We are not witnessing a new scientific revolution“. In: Nordmann, Alfred/Radder, Hans/Schiemann, Gregor (Hrsg.): Science Transformed? Debating Claims of an Epochal Break, Pittsburgh: University of Pittsburgh, S. 31-43.

Silk, Joseph (2006): The Infinite Cosmos: Questions from the Frontiers of Cosmology. Oxford: University Press. 